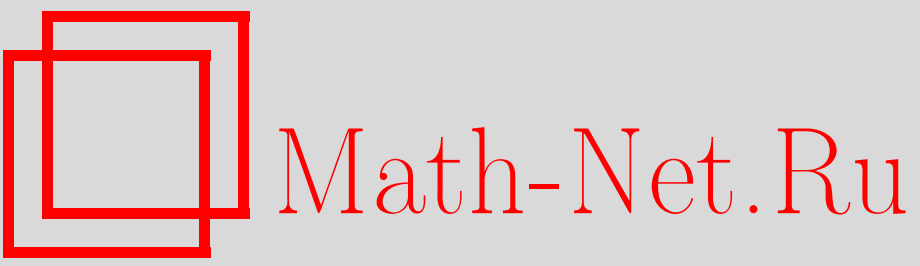

А. А. Гладких, Г. Г. Малинецкий, Нелинейное уравнение Дирака для графена, Матем. моделирование, 2020, том 32, номер 8, 43-56

DOI: https://doi.org/10.20948/mm-2020-08-04

Использование Общероссийского математического портала Math-Net.Ru подразумевает, что вы прочитали и согласны с пользовательским соглашением

http://www.mathnet.ru/rus/agreement

Параметры загрузки:

IP : 3.85 .183 .62

26 апреля 2023 г., 15:33:09 


\title{
НЕЛИНЕЙНОЕ УРАВНЕНИЕ ДИРАКА ДЛЯ ГРАФЕНА
}

\author{
(C) 2020 г. А.А. Гладких ${ }^{1,2}$, Г.Г. Малинецкий ${ }^{1,2}$ \\ ${ }^{1}$ Институт прикладной математики им. М.В. Келдыша РАН \\ ${ }^{2}$ Московский физико-технический институт (национальный исследовательс- \\ кий университет) \\ andrei.gladkikh@phystech.edu; GMalin@Keldysh.ru
}

Работа выполнена при поддержке Российского фонда фундаментальных исследований, проект 19-01-00602 А.

\section{DOI: $10.20948 / \mathrm{mm}-2020-08-04$}

Рассмотрена возможность введения нелинейной поправки в уравнение Дирака для графена с целью адекватного описания коллективных электронных явлений. В отличие от ряда работ по указанной теме, нелинейный член включает в себя не разность, а сумму квадратов компонент спинора. Особое внимание уделено равноправности пространственных координат. Исследованы свойства полученного нелинейного уравнения, претендующего на описание высокотемпературного ферромагнетизма в графене без допущений о ключевой роли дефектов структуры в обеспечении эффекта. Проведено численное моделирование с использованием схемы ЛаксаФридрихса, в результате которого получены сведения о динамике электронной плотности для ряда простейших начальных и краевых условий.

Ключевые слова: нелинейное уравнение Дирака, графен, численное моделирование.

\section{NONLINEAR DIRAC EQUATION FOR GRAPHENE}

\author{
A.A. Gladkikh ${ }^{1,2}$, G.G. Malinetskii ${ }^{1,2}$ \\ ${ }^{1}$ Keldysh Institute of Applied Mathematics (Russian Academy of Sciences) \\ ${ }^{2}$ Moscow Intitute of Physics and Technology (National Research University)
}

We consider a nonlinear Dirac equation in order to describe collective electronic phenomena. In comparison to the other papers on the given topic the interaction term includes the sum of the spinor components' squares instead of their difference. We investigate the properties of the obtained nonlinear equation, pretending to describe hightemperature ferromgnetism without assumptions about the key role of defects. The numerical simulation was done for simple boundary and initial conditions with LaxFriedrichs scheme.

Key words: nonlinear Dirac equation, graphene, numerical simulation. 


\section{1. Введение}

В работе [1] была предпринята попытка объяснить высокотемпературный ферромагнетизм в графене [2] с помощью внесения нелинейной добавки в уравнение Дирака. До этого данные о ферромагнетизме в графене объяснялись влиянием подложки, дефектами структуры или интерпретировались как погрешности измерений.

В работе [1], напротив, с помощью феноменологической поправки изменялась сама основа - уравнение Дирака, а требуемые свойства предлагалось получать как следствия из уравнения, которое было уточнено.

Измененное уравнение в упомянутой работе с помощью ряда приближений сводилось к уравнению Гинзбурга-Ландау-Хиггса, которое, как известно, допускает решение в виде кинка [3].

В некотором смысле исходное уравнение использовалось для того, чтобы только обосновать кинк. И это действительно решало поставленную задачу (кинк - это не точечный, а протяженный солитон, который в некоторых случаях может рассматриваться как доменная стенка $[4,5])$.

Нас же, однако, в первую очередь интересуют уравнения как таковые, а не решения их упрощений в виде кинка. Данная работа посвящена исследованию другого возможного варианта исходного уравнения.

В приведенном нами выводе уравнения нет отождествления спина с псевдоспином, что характерно для ряда работ по указанной теме. Особое внимание при написании уравнения уделено равноправности пространственных координат (дело в том, что нелинейный член в уравнение Дирака можно ввести по-разному, следствием произвола при выборе этого члена может быть существенная неравноправность $x$ и $y$. Было проведено численное моделирование с помощью варианта схемы Лакса-Фридрихса.

\section{2. Уравнения Дирака и Вейля}

Как известно, свободные электроны в графене ведут себя как безмассовые релятивистские фермионы. Приведенное утверждение означает, что поведение частиц в графене может быть описано с помощью уравнения Вейля (которое, как известно, является релятивистским).

Никакого противоречия с теорией относительности при этом не возникает: в действительности электроны не теряют массу и движутся со скоростью $v_{F}$, которая на два порядка меньше скорости света. Дело заключается в том, что носители заряда в графене подчиняются уравнениям, описывающим движение безмассовых двухкомпонентных релятивистких частиц. Смысл компонент спинора при этом совершенно иной, а в роли скорости света выступает упоминавшаяся $v_{F}$. Вместе с тем, не следует полагать, что 
рассматриваемое сходство оканчивается на совпадении уравнений. Ряд таких эффектов, как туннелирование, рождение швингеровских пар, парадокс Клейна, андреевское отражение, ставших известными вовсе не в связи с графеном, оказывается возможным наблюдать при помощи этого материала $[6,7]$.

В уравнении Дирака фигурируют биспиноры. Количество компонент у $\psi$ равно четырём ( $H_{D}$ - гамильтониан Дирака, размерность $\alpha$-матриц -4$)$.

$$
i \frac{\partial \psi}{\partial t}=\hat{H}_{D} \psi, \quad \hat{H}_{D}=v_{F}\left(\alpha^{j} \hat{p}_{j}+m \beta\right) .
$$

Уравнение Вейля соответствует случаю $m=0$. И фигурируют в нём спиноры (количество компонент у $\psi$ и размерность матриц равны 2). Так, для рассматриваемого случая [7]:

$$
\begin{aligned}
& i \frac{\partial \psi}{\partial t}=\hbar v_{F}\left[\left(\begin{array}{cc}
-1 & 0 \\
0 & 1
\end{array}\right)\left(-i \partial_{x}\right)+\left(\begin{array}{cc}
0 & -i \\
i & 0
\end{array}\right)\left(-i \partial_{y}\right)\right] \psi, \\
& H=-i \hbar v_{F}\left(\begin{array}{cc}
-\partial_{x} & -i \partial_{y} \\
i \partial_{y} & \partial_{x}
\end{array}\right) .
\end{aligned}
$$

\section{3. Переход к нелинейному уравнению}

Попытки добавления нелинейных частей в уравнение Дирака предпринимались давно. Различные варианты были предложены Д.Д. Иваненко [8], М. Солером [9] и другими. Вопрос о том, что именно нужно добавить к линейному уравнению носит характер первостепенной важности. Следует еще раз отметить, что вид поправки полностью определяется нами. Мы специально вводим в уравнение нелинейный член.

Свободная энергия

$$
\begin{aligned}
& \Phi=\psi^{+}(\sigma, p) \psi+J\left(\bar{\psi} \sigma_{3} \psi\right)\left(\bar{\psi} \sigma_{3} \psi\right), \\
& \bar{\psi}=\psi^{+} \sigma_{3}, \quad \bar{\psi} \sigma_{3} \psi=\psi^{+} \sigma_{3} \sigma_{3} \psi=\psi^{+} \psi, J-\text { константа связи. }
\end{aligned}
$$

Проварьируем $\Phi$ :

$$
\begin{aligned}
& \frac{\delta \Phi}{\delta \psi^{+}}=(\sigma, p) \psi+2 J \psi^{+} \psi \sigma_{3} \sigma_{3} \psi, \\
& \sigma=\left(\sigma_{1}, \sigma_{2}\right), \quad \sigma_{3}=\left(\begin{array}{cc}
1 & 0 \\
0 & -1
\end{array}\right) .
\end{aligned}
$$

Получаем уравнение 


$$
v_{F}(\sigma, p) \psi+J \psi\left(\psi^{+} \psi\right)=0 .
$$

Один из самых проблематичных моментов связан с переходом к спиновой плотности. Дело заключается в том, что изначально (при выводе уравнения Дирака для графена) рассматривался не спин, а псевдоспин: необходимо было учесть влияние двух взаимопроникающих решеток на свободные электроны. Таким образом, компоненты спинора имеют другую природу и не связаны напрямую с настоящим спином.

В [1] рассматривалась величина $\psi_{1}^{2}-\psi_{2}^{2}$. Поясним, откуда берется знак «-». Пусть $\psi-$ спинор, составленный из компонент $\psi_{1}$ и $\psi_{2}$. Тогда

$$
\psi^{+} \sigma_{3} \psi=\psi_{1}^{2}-\psi_{2}^{2} \text {. }
$$

По-видимому, псевдоспин рассматривался как настоящий спин. Возможно, что есть соображения, оправдывающие подобную операцию (возможно и то, что было рассмотрено нечто совсем другое). Однако, в силу неочевидности перехода, мы не будем в настоящей работе рассматривать спиновую плотность и ограничимся рассмотрением плотности электронной. Принимая $\psi_{k}=a_{k}+i b_{k}$, получаем, что в явном виде интересующее нас уравнение выглядит так:

$$
\left\{\begin{array}{l}
\frac{\partial a_{1}}{\partial t}=\left(\frac{\partial a_{1}}{\partial x}-\frac{\partial b_{2}}{\partial y}\right)-b_{1}\left(a_{1}^{2}+b_{1}^{2}+a_{2}^{2}+b_{2}^{2}\right), \\
\frac{\partial b_{1}}{\partial t}=\left(\frac{\partial b_{1}}{\partial x}+\frac{\partial a_{2}}{\partial y}\right)+a_{1}\left(a_{1}^{2}+b_{1}^{2}+a_{2}^{2}+b_{2}^{2}\right), \\
\frac{\partial a_{2}}{\partial t}=\left(-\frac{\partial a_{2}}{\partial x}+\frac{\partial b_{1}}{\partial y}\right)-b_{2}\left(a_{1}^{2}+b_{1}^{2}+a_{2}^{2}+b_{2}^{2}\right), \\
\frac{\partial b_{2}}{\partial t}=\left(-\frac{\partial b_{2}}{\partial x}-\frac{\partial a_{1}}{\partial y}\right)+a_{2}\left(a_{1}^{2}+b_{1}^{2}+a_{2}^{2}+b_{2}^{2}\right) .
\end{array}\right.
$$

\section{4. Динамическая система}

Без пространственной части система принимает следующий вид:

$$
\left\{\begin{array}{l}
\dot{a}_{1}=-b_{1}\left(a_{1}^{2}+b_{1}^{2}+a_{2}^{2}+b_{2}^{2}\right), \\
\dot{b}_{1}=a_{1}\left(a_{1}^{2}+b_{1}^{2}+a_{2}^{2}+b_{2}^{2}\right), \\
\dot{a}_{2}=-b_{2}\left(a_{1}^{2}+b_{1}^{2}+a_{2}^{2}+b_{2}^{2}\right), \\
\dot{b}_{2}=a_{2}\left(a_{1}^{2}+b_{1}^{2}+a_{2}^{2}+b_{2}^{2}\right) .
\end{array}\right.
$$


Мы имеем дело с системой обыкновенных дифференциальных уравнений. Заметим, что $\frac{d}{d t}\left(a_{i}^{2}+b_{i}^{2}\right)=2\left(a_{i} \dot{a}_{i}+b_{i} \dot{b}_{i}\right)=0$. Таким образом, $a_{1}^{2}+b_{1}^{2}$ и $a_{2}^{2}+b_{2}^{2}$ - первые интегралы системы. Выражение $a_{1}^{2}+b_{1}^{2}+a_{2}^{2}+b_{2}^{2}$ не зависит от времени $t$.

Заметим также, что, продифференцировав каждое уравнение, входящее в систему, получим

$$
\ddot{a}=-C a, \quad \ddot{b}=-C b, \quad \ddot{a}=-C a, \quad \ddot{b}=-C b,
$$

где в качестве $C$ выступает константа, определяемая из начальных условий.

$$
C=\left(a_{1}(0)^{2}+b_{1}(0)^{2}+a_{2}(0)^{2}+b_{2}(0)^{2}\right)^{2} .
$$

Вспомним, как выглядело наше уравнение в комплексном виде:

$$
\left\{\begin{array}{l}
\frac{\partial \psi_{1}}{\partial t}=\frac{\partial \psi_{1}}{\partial x}+i \frac{\partial \psi_{2}}{\partial y}+i\left(\left|\psi_{1}\right|^{2}+\left|\psi_{2}\right|^{2}\right) \psi_{1}, \\
\frac{\partial \psi_{2}}{\partial t}=-\frac{\partial \psi_{2}}{\partial x}-i \frac{\partial \psi_{1}}{\partial y}+i\left(\left|\psi_{1}\right|^{2}+\left|\psi_{2}\right|^{2}\right) \psi_{2} .
\end{array}\right.
$$

Отбрасывая пространственную часть, получаем:

$$
\left\{\begin{array}{l}
\dot{\psi}_{1}=i\left(\left|\psi_{1}\right|^{2}+\left|\psi_{2}\right|^{2}\right) \psi_{1}, \\
\dot{\psi}_{2}=i\left(\left|\psi_{1}\right|^{2}+\left|\psi_{2}\right|^{2}\right) \psi_{2} .
\end{array}\right.
$$

$\left|\psi_{i}\right|$ есть не что иное, как $a_{i}^{2}+b_{i}^{2}$, и $\left|\psi_{1}\right|^{2}+\left|\psi_{2}\right|^{2}$ является постоянной величиной. Таким образом,

$$
\begin{aligned}
& \left\{\begin{array}{l}
\dot{\psi}_{1}=i C \psi_{1}, \\
\dot{\psi}_{2}=i C \psi_{2},
\end{array}\right. \\
& \psi_{i}=\left(a_{i}(0)+b_{i}(0) i\right) \exp (i C t) .
\end{aligned}
$$

Или $\psi_{i}=\sqrt{a_{i}^{2}(0)+b_{i}^{2}(0)} \exp \left(i C t+i \arccos \left(a_{i}(0) / \sqrt{a_{i}^{2}(0)+b_{i}^{2}(0)}\right)\right)$. Так,

$$
\left\{\begin{array}{l}
a_{i}=\sqrt{I_{1}} \cos \gamma_{i}, \\
b_{i}=\sqrt{I_{2}} \sin \gamma_{i},
\end{array}\right.
$$

где $\gamma_{i}=\left(I_{1}+I_{2}\right) t+\arccos \left(a_{i}(0) / \sqrt{I_{i}}\right)$, а $I_{i}=a_{i}^{2}(0)+b_{i}^{2}(0)$.

На рис.1-3 представлено точное решение для системы без пространственной части для начальных условий: $a_{1}(0)=\exp \left(-x^{2}\right), a_{2}(0)=0, b_{1,2}(0)=0$. 

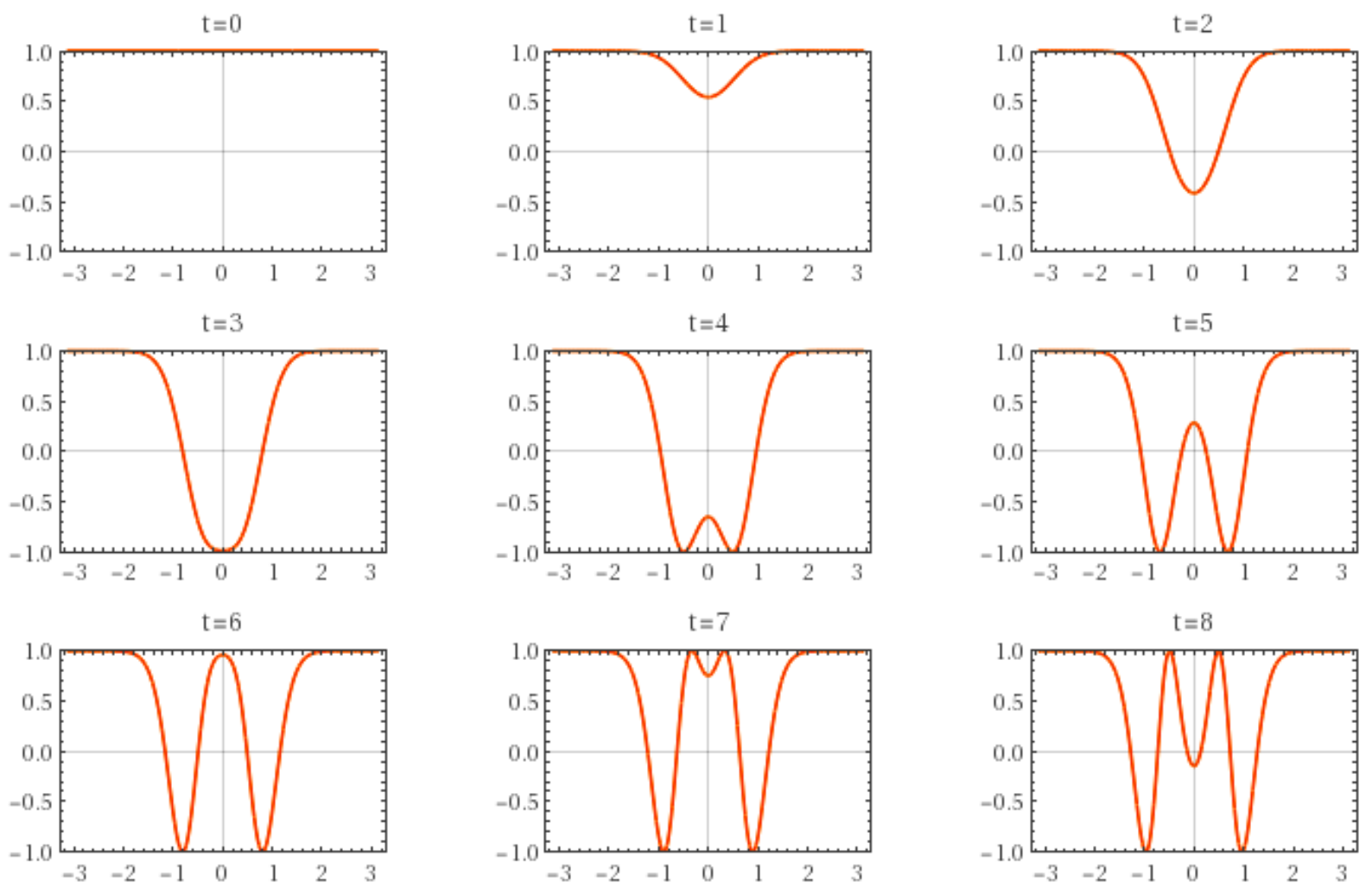

Рис.1. Точное решение для $a_{1}$ при $a_{1}(0)=\exp \left(-x^{2}\right), a_{2}(0)=0, b_{1,2}(0)=0$.
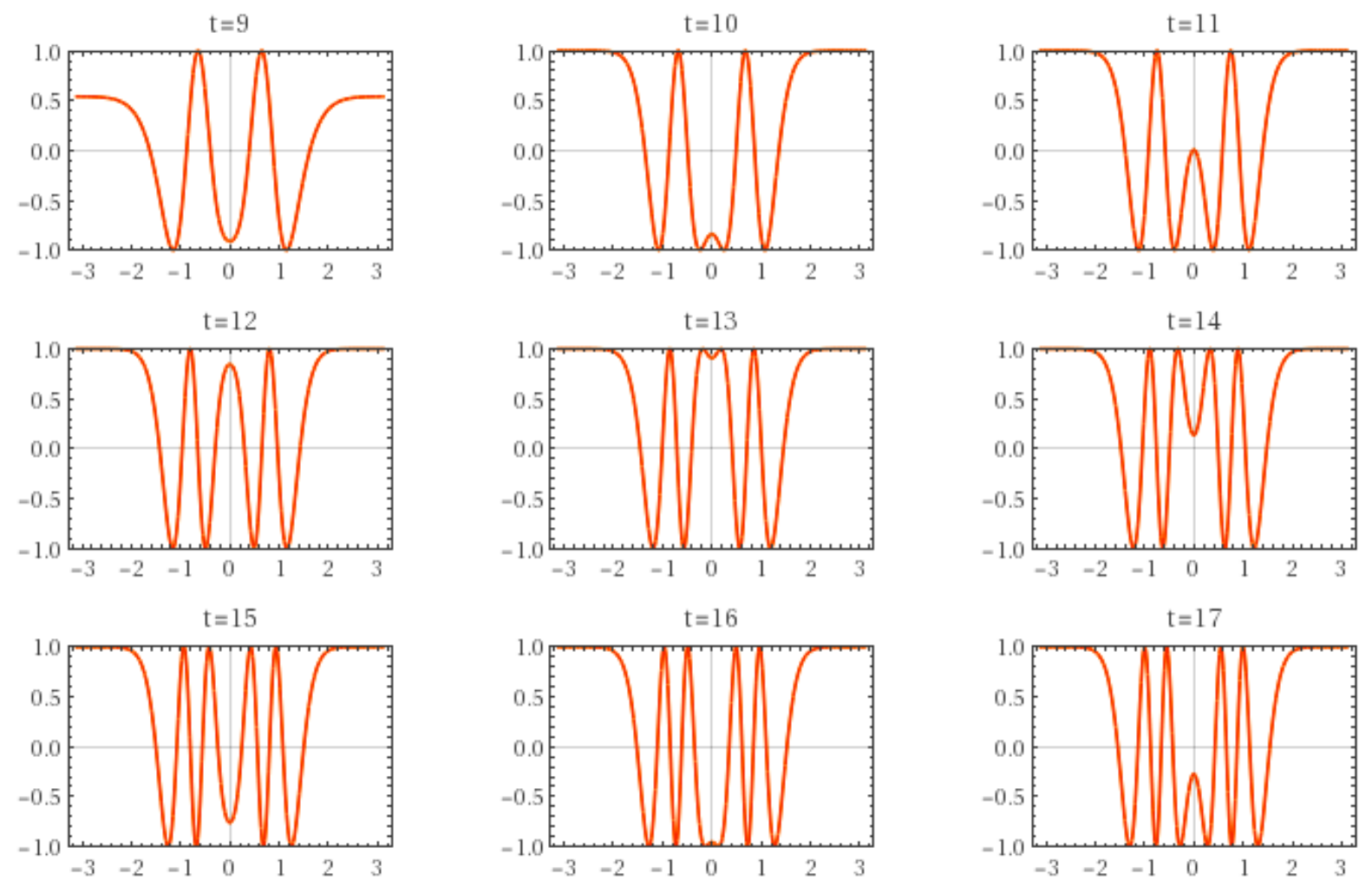

Рис.2. Точное решение для $a_{1}$ при $a_{1}(0)=\exp \left(-x^{2}\right), a_{2}(0)=0, b_{1,2}(0)=0$. 

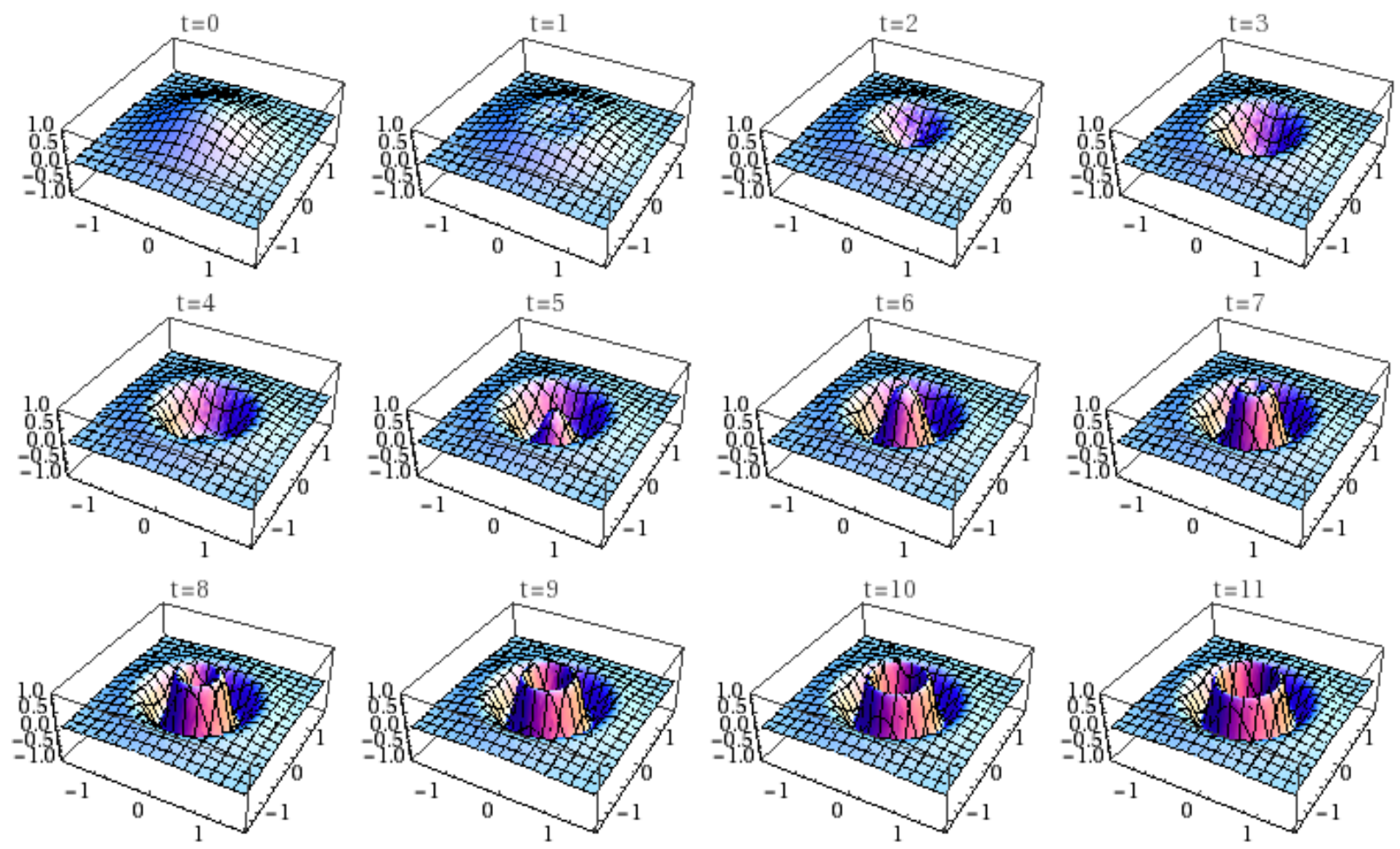

Рис.3. Точное решение системы без пространственной части в $2 D$.

\section{5. Система с пространственной частью}

$I_{1}$ и $I_{2}$ вовсе не будут являться интегралами при добавлении к рассмотренной системе пространственной части. Однако если добавить только зависимость по переменной $x$, то $I_{1}$ и $I_{2}$ останутся сохраняющимися. Действительно, пусть

$$
\left\{\begin{array}{l}
\frac{\partial a_{1}}{\partial t}=\frac{\partial a_{1}}{\partial x}-b_{1}\left(a_{1}^{2}+b_{1}^{2}+a_{2}^{2}+b_{2}^{2}\right), \\
\frac{\partial b_{1}}{\partial t}=\frac{\partial b_{1}}{\partial x}+a_{1}\left(a_{1}^{2}+b_{1}^{2}+a_{2}^{2}+b_{2}^{2}\right), \\
\frac{\partial a_{2}}{\partial t}=-\frac{\partial a_{2}}{\partial x}-b_{2}\left(a_{1}^{2}+b_{1}^{2}+a_{2}^{2}+b_{2}^{2}\right) \\
\frac{\partial b_{2}}{\partial t}=-\frac{\partial b_{2}}{\partial x}+a_{2}\left(a_{1}^{2}+b_{1}^{2}+a_{2}^{2}+b_{2}^{2}\right)
\end{array}\right.
$$

Тогда

$$
\begin{aligned}
& \left(\frac{\partial}{\partial t}-\frac{\partial}{\partial x}\right)\left(a_{1}^{2}+b_{1}^{2}\right)=2 a_{1}\left(\frac{\partial a_{1}}{\partial t}-\frac{\partial a_{1}}{\partial x}\right)+2 b_{1}\left(\frac{\partial b_{1}}{\partial t}-\frac{\partial b_{1}}{\partial x}\right)=0 \\
& \left(\frac{\partial}{\partial t}+\frac{\partial}{\partial x}\right)\left(a_{2}^{2}+b_{2}^{2}\right)=2 a_{2}\left(\frac{\partial a_{2}}{\partial t}+\frac{\partial a_{2}}{\partial x}\right)+2 b_{2}\left(\frac{\partial b_{2}}{\partial t}+\frac{\partial b_{2}}{\partial x}\right)=0
\end{aligned}
$$


Итак,

$$
\begin{aligned}
& \left\{\begin{array}{l}
\left(\frac{\partial}{\partial t}-\frac{\partial}{\partial x}\right)\left(a_{1}^{2}+b_{1}^{2}\right)=0, \\
\left(\frac{\partial}{\partial t}+\frac{\partial}{\partial x}\right)\left(a_{2}^{2}+b_{2}^{2}\right)=0
\end{array}\right. \\
& \left\{\begin{array}{l}
\left(a_{1}^{2}+b_{1}^{2}\right)=f(x+t), \\
\left(a_{2}^{2}+b_{2}^{2}\right)=g(x-t) .
\end{array}\right.
\end{aligned}
$$

Однако в случае с переменной $y$ дело обстоит не так просто. Добавив зависимость по $y$ и убрав зависимость по $x$, получим

$$
\left\{\begin{array}{l}
\partial a_{1} / \partial t=-\partial b_{2} / \partial y-b_{1}\left(a_{1}^{2}+b_{1}^{2}+a_{2}^{2}+b_{2}^{2}\right) \\
\partial b_{1} / \partial t=\partial a_{2} / \partial y+a_{1}\left(a_{1}^{2}+b_{1}^{2}+a_{2}^{2}+b_{2}^{2}\right) \\
\partial a_{2} / \partial t=\partial b_{1} / \partial y-b_{2}\left(a_{1}^{2}+b_{1}^{2}+a_{2}^{2}+b_{2}^{2}\right) \\
\partial b_{2} / \partial t=-\partial a_{1} / \partial y+a_{2}\left(a_{1}^{2}+b_{1}^{2}+a_{2}^{2}+b_{2}^{2}\right)
\end{array}\right.
$$

Покажем, как свести эту систему к требуемому виду

$$
\begin{aligned}
& \left\{\begin{array}{l}
\partial\left(a_{1}+b_{2}\right) / \partial t=-\partial\left(a_{1}+b_{2}\right) / \partial y-\left(b_{1}-a_{2}\right)\left(a_{1}^{2}+b_{1}^{2}+a_{2}^{2}+b_{2}^{2}\right), \\
\partial\left(b_{1}+a_{2}\right) / \partial t=\partial\left(b_{1}+a_{2}\right) / \partial y+\left(a_{1}-b_{2}\right)\left(a_{1}^{2}+b_{1}^{2}+a_{2}^{2}+b_{2}^{2}\right), \\
\partial\left(a_{1}-b_{2}\right) / \partial t=\partial\left(a_{1}-b_{2}\right) / \partial y-\left(b_{1}+a_{2}\right)\left(a_{1}^{2}+b_{1}^{2}+a_{2}^{2}+b_{2}^{2}\right), \\
\partial\left(b_{1}-a_{2}\right) / \partial t-\partial\left(b_{1}-a_{2}\right) / \partial y+\left(a_{1}+b_{2}\right)\left(a_{1}^{2}+b_{1}^{2}+a_{2}^{2}+b_{2}^{2}\right) .
\end{array}\right. \\
& u_{1}=a_{1}-b_{2}, \quad v_{1}=b_{1}+a_{2}, \quad u_{2}=a_{1}+b_{2}, \quad v_{2}=b_{1}-a_{2}, \\
& \left\{\begin{array}{l}
\partial u_{1} / \partial t=\partial u_{1} / \partial y-v_{1}\left(u_{1}^{2}+v_{1}^{2}+u_{2}^{2}+v_{2}^{2}\right) \\
\partial v_{1} / \partial t=\partial v_{1} / \partial y+u_{1}\left(u_{1}^{2}+v_{1}^{2}+u_{2}^{2}+v_{2}^{2}\right) \\
\partial u_{2} / \partial t=-\partial u_{2} / \partial y-v_{2}\left(u_{1}^{2}+v_{1}^{2}+u_{2}^{2}+v_{2}^{2}\right), \\
\partial v_{2} / \partial t=-\partial v_{2} / \partial y+u_{2}\left(u_{1}^{2}+v_{1}^{2}+u_{2}^{2}+v_{2}^{2}\right) .
\end{array}\right.
\end{aligned}
$$

Как видим, при нашем выборе знаков система с одной переменной $x$ и система с одной переменной $y$ приводятся к одному виду. Однако так сделать удается далеко не всегда.

\section{6. Отсутствие инвариантности}

Покажем, что выбор других знаков при нелинейных слагаемых может привести к неравноправности пространственных координат. 
Рассмотрим систему, отличие которой от предыдущей заключается в том, что общая для всех уравнений системы часть нелинейного слагаемого $S$ равна не $a_{1}^{2}+b_{1}^{2}+a_{2}^{2}+b_{2}^{2}=\psi^{+} \psi$, как раньше, а $a_{1}^{2}+b_{1}^{2}-a_{2}^{2}-b_{2}^{2}=\psi^{+} \sigma_{3} \psi$, а знаки при $b_{1} S$ и $a_{1} S$ изменены на противоположные.

$$
\left\{\begin{array}{l}
\partial a_{1} / \partial t=\left(\partial a_{1} / \partial x-\partial b_{2} / \partial y\right)+b_{1}\left(a_{1}^{2}+b_{1}^{2}-a_{2}^{2}+b_{2}^{2}\right), \\
\partial b_{1} / \partial t=\left(\partial b_{1} / \partial x+\partial a_{2} / \partial y\right)-a_{1}\left(a_{1}^{2}+b_{1}^{2}-a_{2}^{2}+b_{2}^{2}\right), \\
\partial a_{2} / \partial t=\left(-\partial a_{2} / \partial x+\partial b_{1} / \partial y\right)-b_{2}\left(a_{1}^{2}+b_{1}^{2}-a_{2}^{2}+b_{2}^{2}\right), \\
\partial b_{2} / \partial t=\left(-\partial b_{2} / \partial x-\partial a_{1} / \partial y\right)+a_{2}\left(a_{1}^{2}+b_{1}^{2}-a_{2}^{2}+b_{2}^{2}\right) .
\end{array}\right.
$$

Мы все так же можем получить $a_{1}^{2}+b_{1}^{2}=f(x+t)$ и $a_{2}^{2}+b_{2}^{2}=g(x-t)$, если мы оставляем $x$. Действительно,

$$
\begin{aligned}
& \left\{\begin{array}{l}
\partial a_{1} / \partial t=\partial a_{1} / \partial x+b_{1}\left(a_{1}^{2}+b_{1}^{2}-a_{2}^{2}-b_{2}^{2}\right), \\
\partial b_{1} / \partial t=\partial b_{1} / \partial x-a_{1}\left(a_{1}^{2}+b_{1}^{2}-a_{2}^{2}-b_{2}^{2}\right), \\
\partial a_{2} / \partial t=-\partial a_{2} / \partial x-b_{2}\left(a_{1}^{2}+b_{1}^{2}-a_{2}^{2}-b_{2}^{2}\right), \\
\partial b_{2} / \partial t=-\partial b_{2} / \partial x+a_{2}\left(a_{1}^{2}+b_{1}^{2}-a_{2}^{2}-b_{2}^{2}\right),
\end{array}\right. \\
& \left(\frac{\partial}{\partial t}-\frac{\partial}{\partial x}\right)\left(a_{1}^{2}+b_{1}^{2}\right)=2 a_{1}\left(\frac{\partial a_{1}}{\partial t}-\frac{\partial a_{1}}{\partial x}\right)+2 b_{1}\left(\frac{\partial b_{1}}{\partial t}-\frac{\partial b_{1}}{\partial x}\right)=0 .
\end{aligned}
$$

Аналогично, $(\partial / \partial t+\partial / \partial x)\left(a_{2}^{2}+b_{2}^{2}\right)=0$. Таким образом,

$$
\begin{aligned}
& \left\{\begin{array}{l}
(\partial / \partial t-\partial / \partial x)\left(a_{1}^{2}+b_{1}^{2}\right)=0 \\
(\partial / \partial t+\partial / \partial x)\left(a_{2}^{2}+b_{2}^{2}\right)=0
\end{array}\right. \\
& \left\{\begin{array}{l}
\left(a_{1}^{2}+b_{1}^{2}\right)=f(x+t) \\
\left(a_{2}^{2}+b_{2}^{2}\right)=g(x-t)
\end{array}\right.
\end{aligned}
$$

Попытаемся проделать то же для переменной $y$, убрав $x$.

$$
\left\{\begin{array}{l}
\partial a_{1} / \partial t=-\partial b_{2} / \partial y+b_{1}\left(a_{1}^{2}+b_{1}^{2}-a_{2}^{2}-b_{2}^{2}\right) \\
\partial b_{1} / \partial t=\partial a_{2} / \partial y-a_{1}\left(a_{1}^{2}+b_{1}^{2}-a_{2}^{2}-b_{2}^{2}\right) \\
\partial a_{2} / \partial t=\partial b_{1} / \partial y-b_{2}\left(a_{1}^{2}+b_{1}^{2}-a_{2}^{2}-b_{2}^{2}\right) \\
\partial b_{2} / \partial t=-\partial a_{1} / \partial y+a_{2}\left(a_{1}^{2}+b_{1}^{2}-a_{2}^{2}-b_{2}^{2}\right)
\end{array}\right.
$$

Мы можем проделать следующие операции, которые не приводят к желаемому результату, а наоборот, показывают степень различия между переменными. 


$$
\begin{aligned}
& (\partial / \partial t+\partial / \partial x)\left(a_{1}^{2}-b_{2}^{2}\right)=2 a_{1}\left(\partial a_{1} / \partial t+\partial a_{1} / \partial y\right)-2 b_{2}\left(\partial b_{2} / \partial t+\partial b_{2} / \partial y\right)= \\
& =a_{1}\left(\partial a_{1} / \partial t+a_{2} \mathbf{S}-\partial b_{2} / \partial t\right)-b_{2}\left(\partial b_{2} / \partial t+b_{1} \mathbf{S}-\partial a_{1} / \partial t\right)= \\
& =\left(a_{1} a_{2}-b_{1} b_{2}\right)\left(a_{1}^{2}+b_{1}^{2}-a_{2}^{2}-b_{2}^{2}\right)+\left(a_{1}+b_{2}\right)\left(\partial a_{1} / \partial t-\partial b_{2} / \partial t\right) \\
& (\partial / \partial t-\partial / \partial x)\left(b_{1}^{2}-a_{2}^{2}\right)=2 b_{1}\left(\partial b_{1} / \partial t-\partial b_{1} / \partial y\right)-2 a_{2}\left(\partial a_{2} / \partial t-\partial a_{2} / \partial y\right)= \\
& =b_{1} \partial b_{1} / \partial t-a_{2} \partial a_{2} / \partial t-b_{1} \partial a_{2} / \partial t-b_{1} b_{2} \mathbf{S}+a_{2} \partial b_{1} / \partial t+a_{1} a_{2} \mathbf{S}= \\
& =\left(a_{1} a_{2}-b_{1} b_{2}\right)\left(a_{1}^{2}+b_{1}^{2}-a_{2}^{2}-b_{2}^{2}\right)+\partial a_{1} / \partial t\left(a_{1}+b_{2}\right)= \\
& =\left(a_{1} a_{2}-b_{1} b_{2}\right)\left(a_{1}^{2}+b_{1}^{2}-a_{2}^{2}-b_{2}^{2}\right)+\left(a_{2}+b_{1}\right)\left(\partial b_{1} / \partial t-\partial a_{2} / \partial t\right)
\end{aligned}
$$

Может показаться, что свести систему к исходной поможет следующая замена:

$$
u_{1}=a_{1}+b_{2}, \quad u_{2}=a_{1}-b_{2}, \quad v_{1}=b_{1}+a_{2}, \quad v_{2}=b_{1}-a_{2} .
$$

Действительно,

$$
\left\{\begin{array}{l}
\partial\left(a_{1}+b_{2}\right) / \partial t=-\partial\left(a_{1}+b_{2}\right) / \partial y+\left(b_{1}+a_{2}\right)\left(a_{1}^{2}+b_{1}^{2}-a_{2}^{2}-b_{2}^{2}\right) \\
\partial\left(b_{1}+a_{2}\right) / \partial t=\partial\left(b_{1}+a_{2}\right) / \partial y-\left(a_{1}+b_{2}\right)\left(a_{1}^{2}+b_{1}^{2}-a_{2}^{2}-b_{2}^{2}\right) \\
\partial\left(a_{1}-b_{2}\right) / \partial t=\partial\left(a_{1}-b_{2}\right) / \partial y+\left(b_{1}-a_{2}\right)\left(a_{1}^{2}+b_{1}^{2}-a_{2}^{2}-b_{2}^{2}\right) \\
\partial\left(b_{1}-a_{2}\right) / \partial t=-\partial\left(b_{1}-a_{2}\right) / \partial y-\left(a_{1}-b_{2}\right)\left(a_{1}^{2}+b_{1}^{2}-a_{2}^{2}-b_{2}^{2}\right)
\end{array}\right.
$$

Однако в новых переменных уравнения приобретают вид

$$
\left\{\begin{array}{l}
\partial u_{1} / \partial t=-\partial u_{1} / \partial y+v_{1}\left(u_{1} u_{2}+v_{1} v_{2}\right) \\
\partial v_{1} / \partial t=\partial v_{1} / \partial y-u_{1}\left(u_{1} u_{2}+v_{1} v_{2}\right) \\
\partial u_{2} / \partial t=\partial u_{2} / \partial y+v_{2}\left(u_{1} u_{2}+v_{1} v_{2}\right) \\
\partial v_{2} / \partial t=-\partial v_{2} / \partial y-u_{2}\left(u_{1} u_{2}+v_{1} v_{2}\right)
\end{array}\right.
$$

Ясно, что эти уравнения не эквивалентны уравнениям, полученным для переменной $x$. Рассмотрим также, что будет, если выключить нелинейность и убрать правую часть. В случае задания начального значения только для переменной $b_{1}$ получим

$$
\begin{aligned}
& \omega_{j}=(-1)^{j} \sqrt{k_{x}^{2}+k_{y}^{2}}, \quad j=1,2, \\
& \left\{\begin{array}{l}
a_{i}=A_{1} \cos \left(k_{x} x+k_{y} y+\sqrt{k_{x}^{2}+k_{y}^{2}} t\right) \\
b_{i}=A_{2}\left(\left(k_{x}+(-1)^{i+j} \sqrt{k_{x}^{2}+k_{y}^{2}}\right) / k_{y}\right)\left(\cos \left(k_{x} x+k_{y} y+\sqrt{k_{x}^{2}+k_{y}^{2}} t\right)\right) .
\end{array}\right.
\end{aligned}
$$




\section{7. Численный эксперимент}

Заметим, что наша система допускает представление в виде $\mathbf{u}_{t}=A \mathbf{u}_{x}+B \mathbf{u}_{y}+\mathbf{f}$, где $\psi_{k}=a_{k}+i b_{k}$,

$$
\begin{aligned}
& A=\left(\begin{array}{cccc}
1 & 0 & 0 & 0 \\
0 & 1 & 0 & 0 \\
0 & 0 & -1 & 0 \\
0 & 0 & 0 & -1
\end{array}\right), \quad B=\left(\begin{array}{cccc}
0 & 0 & 0 & -1 \\
0 & 0 & 1 & 0 \\
0 & 1 & 0 & 0 \\
-1 & 0 & 0 & 0
\end{array}\right), \\
& \mathbf{u}=\left(\begin{array}{l}
a_{1} \\
b_{1} \\
a_{2} \\
b_{2}
\end{array}\right), \quad \mathbf{f}=\left(\begin{array}{cccc}
0 & -1 & 0 & 0 \\
1 & 0 & 0 & 0 \\
0 & 0 & 0 & -1 \\
0 & 0 & 1 & 0
\end{array}\right) \mathbf{u}^{*} \mathbf{u}^{T} \mathbf{u} .
\end{aligned}
$$

У нас имеются три переменные: $x, y, t$. Существует целый ряд определений гиперболичности многомерных систем (по Петровскому, Фридрихсу и т.д.). Нам важно, чтобы линейная замена пространственных координат не меняла сам тип процесса. Поэтому условие гиперболичности будем накладывать на все матрицы вида $\alpha A+\beta B$, где $\alpha^{2}+\beta^{2} \neq 0$ (рассматривается уравнение $\left.\mathbf{u}_{t}=A \mathbf{u}_{x}+B \mathbf{u}_{y}\right)$.

Вспоминая вид матриц $A$ и $B$, получаем вид $\alpha A+\beta B$ :

$$
\alpha\left(\begin{array}{cccc}
1 & 0 & 0 & 0 \\
0 & 1 & 0 & 0 \\
0 & 0 & -1 & 0 \\
0 & 0 & 0 & -1
\end{array}\right)+\beta\left(\begin{array}{cccc}
0 & 0 & 0 & -1 \\
0 & 0 & 1 & 0 \\
0 & 1 & 0 & 0 \\
-1 & 0 & 0 & 0
\end{array}\right)=\left(\begin{array}{cccc}
\alpha & 0 & 0 & -\beta \\
0 & \alpha & \beta & 0 \\
0 & \beta & -\alpha & 0 \\
-\beta & 0 & 0 & -\alpha
\end{array}\right) .
$$

Определитель матрицы, составленной из собственных векторов будет больше нуля для $\forall(\alpha, \beta): \alpha^{2}+\beta^{2} \neq 0$

$$
\operatorname{det}\left(\begin{array}{cccc}
-\left(\alpha-\sqrt{\alpha^{2}+\beta^{2}}\right) / \beta & 0 & 0 & 1 \\
0 & -\left(\sqrt{\alpha^{2}+\beta^{2}}-\alpha\right) / \beta & 1 & 0 \\
-\left(\alpha+\sqrt{\alpha^{2}+\beta^{2}}\right) / \beta & 0 & 0 & 1 \\
0 & -\left(-\alpha-\sqrt{\alpha^{2}+\beta^{2}}\right) / \beta & 1 & 0
\end{array}\right)=\frac{4 \alpha^{2} \beta^{2}+4 \beta^{4}}{\beta^{4}} \geq 0 .
$$


Плоскость $O x y$ - плоскость графена. По оси аппликат откладывается величина $\psi^{+} \psi$. Заметим, что она целиком входит в правую часть каждого из уравнений системы.

Величина $\psi^{+} \psi$ никак не связана с нормировкой, что несколько непривычно, $\psi$ является спинором, а $\psi^{+} \psi$ имеет смысл плотности вероятности.

Динамика плотности вероятности $\psi^{+} \psi$, полученная в результате численного моделирования системы (9) с использованием варианта схемы Лакса-Фридрихса для $a_{1}(0)=\exp \left(-3\left(x^{2}+y^{2}\right)\right), a_{2}(0)=0, b_{1,2}(0)=0$ и нулевых граничных условий представлена на рис.4. Характерная динамика системы для случая с отождествлением противоположных границ рассматриваемой области представлена на рис.5.
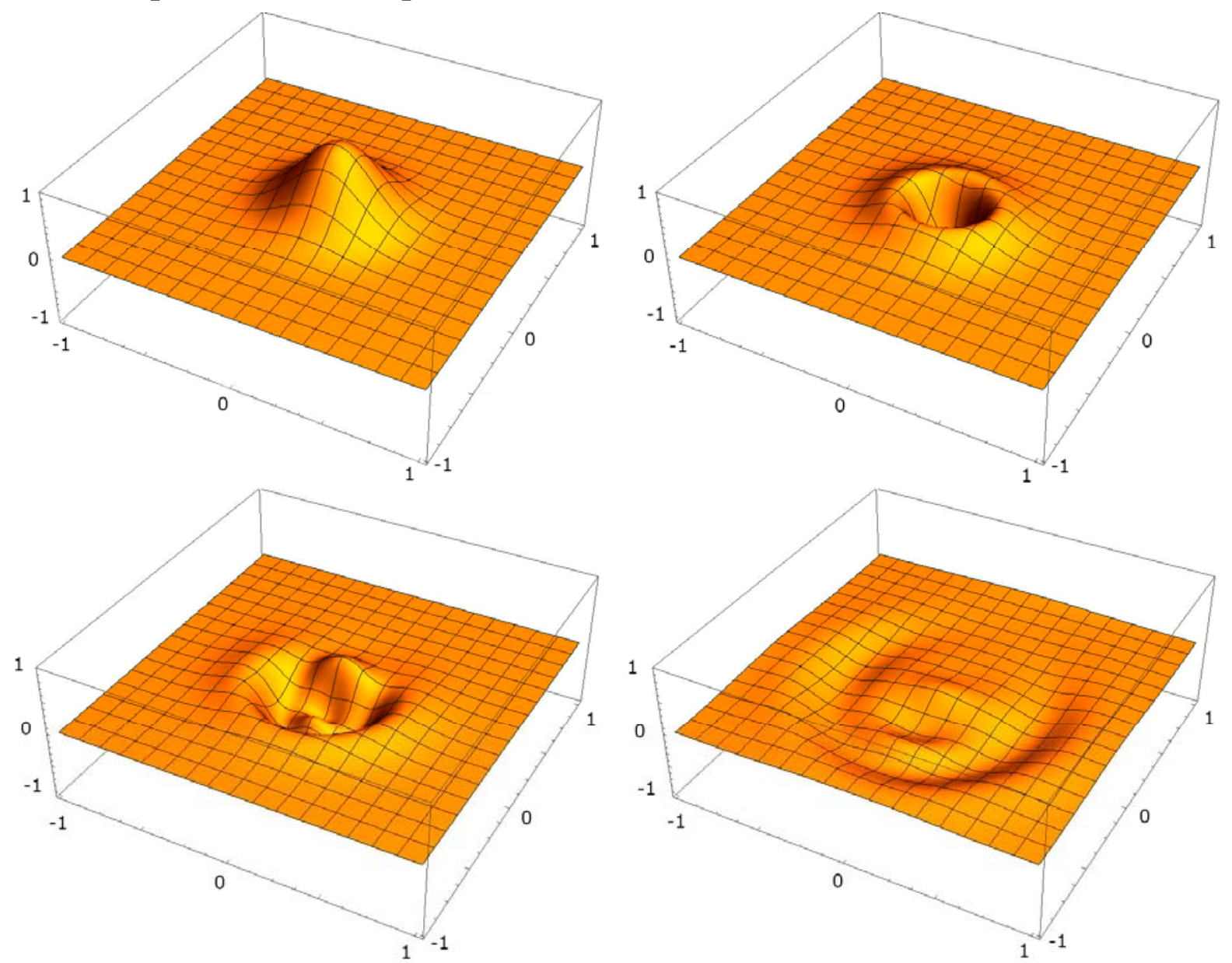

Рис.4. Динамика $\psi^{+} \psi$ при $a_{1}(0)=\exp \left(-10\left(x^{2}+y^{2}\right)\right), a_{2}(0)=0, b_{1,2}(0)=0$, $\left.\psi^{+} \psi\right|_{x=-1}=\left.\psi^{+} \psi\right|_{y=-1}=\left.\psi^{+} \psi\right|_{x=1}=\left.\psi^{+} \psi\right|_{y=1}=0$.

\section{8. Заключение}

В замкнутой форме выписано уравнение, претендующее на описание электронных явлений в графене, обобщающее уравнение Дирака. Рассмот- 
рены системы, получающиеся путем упрощения исходной, также выписаны решения для соответствующих систем.
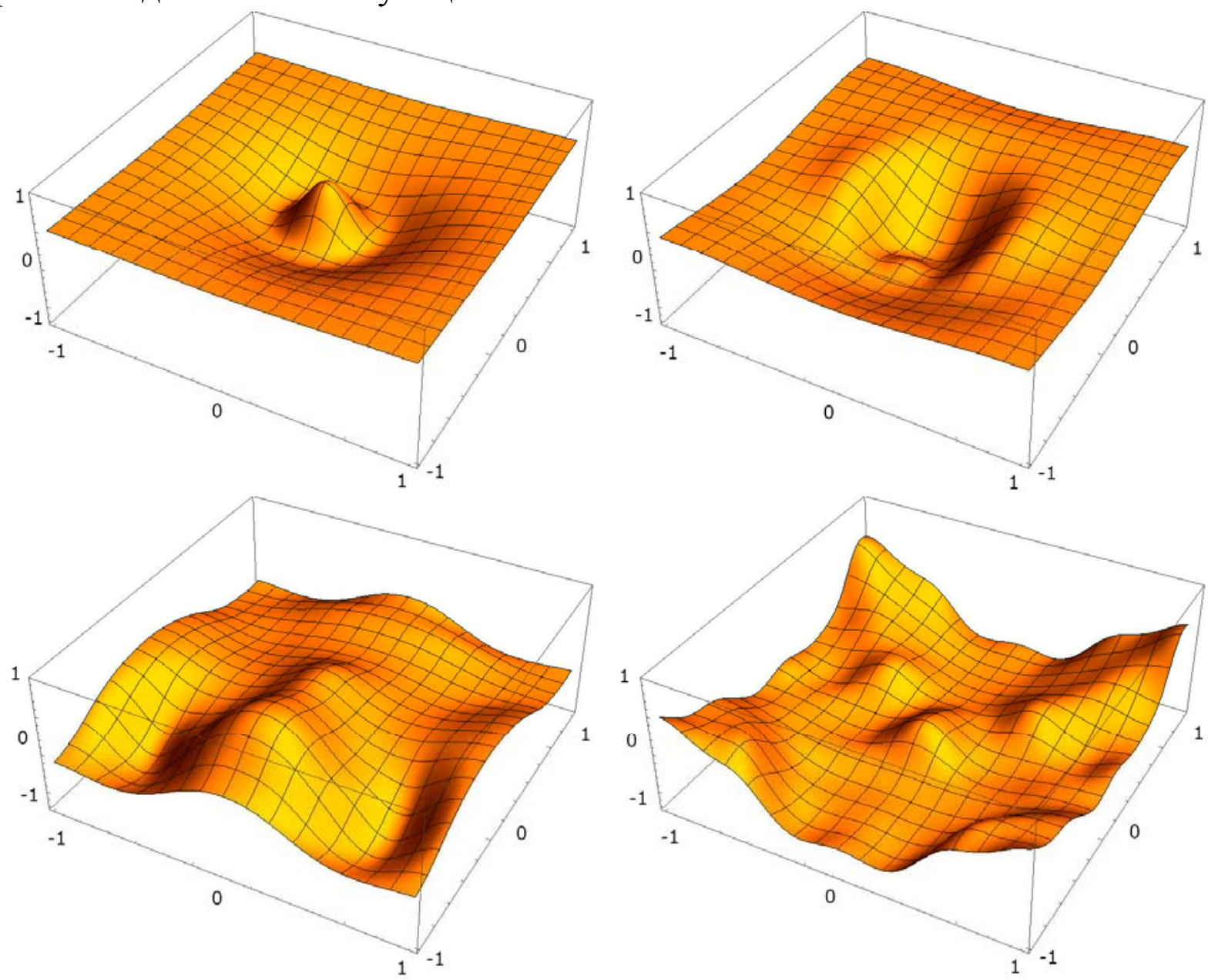

Рис.5. Характерная динамика $\psi^{+} \psi$ при отождествлении противоположных границ расчетной области.

Проведено моделирование системы с помощью схемы Лакса-Фридрихса для ряда простейших начальных условий. Было показано, что в случае включения как нелинейной, так и пространственной частей уравнения, изначальное возмущение распадается, а возникновения колебаний, характерных для случая с одной нелинейной частью, не происходит.

Было также продемонстрировано, что динамика системы в случае периодических краевых условий может быть довольно сложна. Численные эксперименты при этом носят предварительный характер и призваны продемонстрировать, что полученные уравнения богаты и содержательны.

Анализ свойств упрощенных систем представляется важным для того, чтобы понять, какие эффекты были характерны уже для отдельных частей, а какие могут быть достигнуты только за счет сочетания составляющих.

Поиск подобных нетривиальных эффектов является задачей для будущего. Хочется верить, что эта задача будет интересна с общематематичес- 
кой точки зрения, а не только в связи с возможностью описания электронных явлений.

Авторы считают своим долгом выразить признательность Д.Д. Грачеву, который привлек их внимание к интересной задаче. Авторы благодарят Ф. Попова за обсуждение текста статьи.

\section{СПИСОК ЛИТЕРАТУРЫ}

1. Derbov V.L. et al. Model for spin waves and lasing in monolayer graphene films // Saratov Fall Meeting 2014. - International Society for Optics and Photonics, 2015.

2. Wang Y. et al. Room-temperature ferromagnetism of graphene // Nano letters, 2008, v.9, № 1, c.220-224.

3. Белова Т.И., Кудрявиев А.Е. Солитоны и их взаимодействия в классической теории поля // Успехи физ. наук, 1997, т.167, № 4, с.377-406;

Belova T.I., Kudryavtsev A.E. Solitons and their interactions in classical field theory // Physics-Uspekhi, 1997, v. 40, № 4, p. 359.

4. Vachaspati T. Kinks and domain walls: An introduction to classical and quantum solitons. - Cambridge University Press, 2006.

5. Грачёв Д.Д. и др. Ферромагнетизм в графеновых и фуллереновых наноструктурах. Теория, моделирование, эксперимент // Вестник РУДН. Серия «Математика, информатика, физика, 2010, т.1, с.22-27;

Grachyov D.D. $i$ dr. Ferromagnetizm v grafenovyh i fullerenovyh nanostrukturah. Teoriya, modelirovanie, eksperiment // Vestnik RUDN. Seriya «Matematika, informatika, fizika», 2010, t.1, s.22-27.

6. Лозовик Ю.Е., Меркулова С.П., Соколик А.А. Коллективные электронные явления в графене // Успехи физических наук, 2008, т.178, № 7, с.757-776;

англ. пер.: Lozovik Y.E. et al. Collective electron phenomena and electron transport in graphene Scientific Session of the Physical Sciences Division of the Russian Academy Of Sciences (27 February 2008) // Physics-Uspekhi, 2008, т.51, № 7, c.727.

7. Маслов В. Свойства графена и (2+1)-мерное уравнение Дирака. - М.: МГУ им. М.В. Ломоносова, 2015.

Maslov V. Svojstva grafena i mernoe uravnenie Diraka. - M.: MGU im. M.V. Lomonosova.

8. Иваненко Д.Д. Замечание о теории взаимодействия через частицы // ЖЭТФ, 1938, т.8, с.260-266;

Ivanenko D.D. Zamechanie o teorii vzaimodejstviya cherez chasticy // ZHETF, 1938, t.8, c.260-266.

9. Soler M. Classical, stable, nonlinear spinor field with positive rest energy // Physical Review D., 1970, т.1, № 10, c.2766.

10. Akhmedov E.T., Popov F.K., Slepukhin V.M. Infrared dynamics of the massive $\varphi^{4}$ theory on de Sitter space // Physical Review D., 2013, т.88, № 2, c.024021.

Поступила в редакцию 05.09.2019

После доработки 05.09.2019

Принята к публикации 21.10.2019 